The following works of scientific interest will be published by Messrs. Macmillan and Co., during the coming season :-_"A Treatise on Comparative Embryology," by Mr. F. M. Balfour, F.R.S.; the second part of the second volume of Professors Roscoe and Schorlemmer's "Treatise on Chemistry"; this, which is just ready, completes the "Inorganic Chemistry;" Prof. Boyd Dawkins" "Early Man in Britain"; Prof. Gamgee's "TextBook of the Physiological Chemistry of the Animal Body;" "Pharmacology and Therapeutics" and "Natural History in the Bible," by Dr. Lauder Brunton, F.R.S. ; "A Manual of Geology," by Prof. Geikie, F.R.S. ; "Structural Botany on the Basis of Morphology," by Prof. Asa - Gray; "Blowpipe Analysis," from the German of J. Landauer, by Messrs. James Taylor and W. E. Kay; "Questions on Chemistry," by Mr. Francis Jones; "Easy Lessons on Heat," by Miss C. A. Martineau ; "Easy Lessons on Light," by Mrs. F. E. Arrdry; "A Handbook of Double Stars," with a Catalogue of I,200 Double Stars and Extensive Lists of Measures for the Use of Amateurs, by Edward Crossley, F.R.A.S., Joseph Gledhill, F.R.A.S., and James M. Wilson, F.R.A.S., with Illustrations; and a new and thoroughly revised edition of "Pharmacographia," by Messrs. Fliickiger and Hanbury. Prof. Huxley's "Introductory" to the Science Primers, has already been announced.

THE additions to the Zoological Society's Gardens during the past week inciude a Vervet Monkey (Cercopithecus lalandii) rom South Africa, presented by Mr. E. Meyerstein; a Black Rat (Mus rattus) from Rangoon, presented by Mr. R. M. Middleton; a Norwegian Lemming (Myodes lemmus) from Norway, presented by Mr. James Shuter, F.R.C.S.; a Bonelli's Eagle (Nisaetus fasciatus) from Mogador, presented by Capt. W. P. Forwood; a King Parrakeet (Aprosmictus scapulatus) from New South Wales, presented by General Blake; a Whitebacked Piping Crow (Gymnorhina leuconota) from South Australia, presented by Mrs. Buchanan; a Silky Marmoset (Midas rosalia) from Brazil, a Malbrouck Monkey (Cercopithecus cynosurus) from West Africa, deposited; a Red-billed Tree Duck (Dendrocygna autumnalis) from South America, purchased; a Brown Bear (Ursus arctos) from Russia, received in exchange.

\section{MOUNT ETNA}

GIIORTLY after the eruption of Mount Etna in May and June last M.H. de Saussure visited the mountain, and made a minute exploration of the region about the sources of the eruption. The results of this examination he describes in a series of letters in the formal de Genize from June 17 to July 4, copies of which $M$. de Saussure has sent us, along with a note containing additional observations. On his first ascent he and his guide bad to spend the night on Monte Temperossa in rather critical circumstances and with the scantiest supply of food and drink. Indeed, it seems to have taken a day or two after reaching a supply of water below ere M. de Saussure's thirst could be quenched. $\mathrm{H}$ had observed large patches of a deep black attached to the rocks, and had been puzzled to know what they were. His thirst, however, quickened his perception, and on scratching away about a centimetre of ashes he found underneath what he had half expected, beautiful white snow. The failure of this first excursion determined M. de Saussure to choose another point of departure than Lingugrossa, and to devote three days if necessary to traverse the neighbourhood of the cone. He thus succeeded in reaching the upper limits of the eruption on the north side. The crevasse which has been opened on Etna has divided the volcano into two parts. On the north face it extends to the valley which bounds the mountain ; on the south face it seems to be arrested half way. Some of the details of the eruption have already been given in NaTURE, vol. xx. p. r98. On the rorth face the large crevasse gave out two considerable streams, superposed in the same fault. The upper stream began in the neighbourhood of the cone of Etna, and was arrested to the east of Monte Pernicro. Thence the crevasse is quite exposed, and gave out only eruption of gas. Below Monte Pizzello there is formed a cone of ashes which gave out no lava. From this point the crevasses continue exposed to the ravine which separates Monte Nero from Monte Temperossa. Here are the sources and centres of the great lava stream which extend to near Mojo. M. de Satissure describes in detail the upper stream, which appears not to have been previously visited.

This eruption first appears at a height of about 2,700 metres, at the foot of the slopes of the upper platean which supports the principal cone (Monte Morigibello). A violent explosion has here opened the side of the mountain, throwing out a mass of rocks, and forming a steep gulf in which the northern crevasse seems to terminate. Below this point of explosion the lava was thrown out in great abundance, and formed a current at first narrow, which afterwards inundated the slopes of a high plateau spreading over a distance of several kilometres. At the point of departure was formed a sugar-loaf cone of small height, which, on June $1_{3}$, was still very active, and whence escaped with a hissing sound a thick smoke of vapours, mixed occasionally with great flames resulting from the combustion of gas. The lava was spread over a vast inclined plateau, flowing over very rough streams of recent lava, on which it is broken up to an infinite extent as far as the foot of a mountain with three craters (Monte Pernicio) which turns it to the east.

An important fact observed by $M$. de Saussure is that these lavas flowed over the snow, and that at the time of his visit even they rested on a thick bed of that substance. In fact, in all the faults of the lava, in all the openings, at the bottom of all the ravines resulting from the sinking of the lavas, snow was found, often several metres in thickness. Nothing proves better, $M$. de Saussure thinks, how bad a conductor of heat the eruptive matter is. The terminal end, as it rolls down, carpets the ground with blocks resulting from the continual rupture of the alreacly solidified envelope, and thus forms a base on which afterwards flows the viscous current. At the same time a large mass of snow must have been melted. M. de Saussure saw traces of a large number of streams loaded with ashes which bacl been precipitated from all the rocks and washed the slopes of the snows, which extend much lower than the lava stream. One result of this eruption over snow is that the lava is mixed with a mass of mud, the melting snow diluting the dust resulting from the porphyrisation of the blocks at the same time as the shower of ashes from the great crater falls on the surface of the current. The fire and water ceaselessly intermingling by the mechanical action of the burning gravel, "produced a sort of muddy marmalade, which, rapidly fusing by the persistent heat of the lavas, gave rise to clonds of vapour and left all the stream, all its blocks, all its pebbles, covered with a layer of dry mud, which turns into dust and gives to the new lavas a grey colour which prevents them from being distinguished at a distance from old lavas.

Below the upper lavas the slopes which extend in the direction of Monte Nero are covered with vast fields of hard and deep snow. Their surface is all strewn with yellow spots, so that at a distance they seem covered with sulphur. This appearance is due to the abundant efflorescence of ferrouts chloride which condenses on the surface of the snow, and which has formed small mound-like masses, mixed to a smail extent with agglutinated ashes. The whole of the snow is, moreover, covered with patches of hardened mud of the size of a plate, and even much larger, also ornamented with yellow efflorescence, and mostly cracked like dried mud or split from bottom to top by a push which has raised them and broken them into a star-like shape. M. de Saussure thinks that these singular accidents are due to deep emanations, and seem to show that under the snow the ground is riddled with crevasses in direct communication with the volcanic centre. The acid vapours reaching the lower surface of the snow are there condensed by the cold ; but gradually they reach the surface, and have then formed an infinite number of small sources, which are charged with mud because the ashes of the old subjacent lavas have been diluted with water resulting from the condensation of vapour against the under surface of the snow, increased by the snow melted by the heat of these same vapours. After quitting the snows, an immense area of sand-hills is met with covered with blocks from the crater of Etna.

M. de Saussure paid a visit to the great cone of Etria in company with an English and an American tourist. As they were walking over the lower slopes of the cone of ashes, he observed a small crack in the fine sand which covered the ground. This soon ceased to be visible, and suddenly M. de Saussure saw a much larger crevasse open under his feet, and he felt at the same time the ground begin to glide down the steep slope. He fled 
as fast as he could, and reached a solid standing-ground, but not before perceiving that the guide, who walked behind him, had sunk up to the middle in the sand, and indeed soon disappeared. All the slope sank with increasing swiftness, swelling out at the bottom and opening out to give forth a stream of mud of at least ten metres in height, which shot like a wild torrent across the plateau, and precipitated itself towards the Val de Bove. Immediately all the moving sand, to more than thirty metres in height, was diluted and drawn along, as were also the stones and blocks it contained, and which rolled over each other pell-mell. Fortunately they sticceeded in extricating the guide, covered with mud, torn and bruised.

Seeking the origin of these ermptions of mud, M. de Saussure observes first that they take place in the neighbourhood of the central cone of Etra, and that they all escape from the foot of the cone and the very inclined slopes which support it. They proceed then exclusively from the water stored in the beds of ashes. These causes seem to account for this mass of water. I. The hot vapours. The cone seems almost exclusively com. posed of ashes and movable stones, and during the eraption an enormous mass of vapour must have traversed it. There are, moreover, numerous fumeroles in the upper part. These vapours have been condensed, all the more readily that they have encrusted the mantle of snow which envelops the summit of the mountain. 2 . The melting of this snow, determined less by the fall of ashes on the surface than by the heat which radiates from the interior and the vapours which traverse the porous mass of the cone. 3. The abundant vapours falling from the cloud of vapours projected by the crater. The two first causes are the most important; the last has most especially given rise to surface streams, the traces of which are apparent. The water gradually collecting in excess in the lower parts of the cone, ends by forming these enormous deposits, which, at a given moment, yielding to their weight, cause an eruption to the outside, turning over and diluting the beds of ashes which oppose its exit.

In a communication to us $M$. de Saussure gives an analysis of the little mud-heaps which have projected through the snow. They are composed of a grey powder formed of pozzolana (spongy amorphous silicates), white, grey, and black (augite), mixed with $(b)$ amorphous sulphur (yellow and orange coloured); (c) calcium sulphate (hydrated) in numerous well-defined small crystals; $(d)$ sodium and potassium sulphate; $(e)$ calcium and potassium chloride; $(f)$ perchloride of iron and oxychloride of iron: $(g)$ indication of copper salts.

The crystals are all of alabaster, and so wet that they can only have been formed after the eruption of the mud in drying. There is no crystallised silica nor feldspar. The sulphur in the mud is curious to observe. It must have condensed out of the eruptive vapours which formed the water to make the mud by mixing with old ashes under the snow, and by their expansion driven small quantities of that mud through the snow. This mud, when heated, evaporates sulphur, sulphurous and sulphuric acid, some perchloride of iron, and some hydrochloric acid.

\section{THE ACTION OF HEAT IN VACUO ON $M E T A L S^{1}$}

IN the course of my experiments on electric lighting I have developed some striking phenomena arising from the heating of metals by flames and by the electric current, especially wires of platinum, and platinum alloyed with iridium. These experiments are in progress.

The first fact observed was that platinum lost weight when heated in a flame of hydrogen, that the metal coloured the flame green, and that these two results continued until the whole of the platinum in contact with the flame had disappeared. A platinum wire four-thousandths of an inch in diameter, and weighing 306 mgrms., was bunched together and suspended in a hydrogen flame. It lost weight at the rate of a fraction less than I mgrm. per hour as long as it was suspended in the flame. When a platinum wire is stretched between two clamping posts, and arranged to pass through a hydrogen flame, it is coloured a light green ; but when the temperature of the wire is raised above that of the flame, by passing a current through it, the flame is coloured a deep green. To ascertain the diminution in the weight of a platinum wire when heated by the electric current, I placed between two clamping posts a wire five-thousandths of

A Paper read by Mr. T. A. Edison before the American Association for the Advancement of Science ; Saratoga Meeting. an inch in diameter, and weighing 266 mgrms. This wire, after it was brought to incandescence for twenty minutes by the current, lost I mgrm. The same wire was then raised to incandescence, for twenty minutes it gave a loss of 3 mgrms. Afterwards it was kept incandescent for one hour and ten minutes, at which time it weighed $258 \mathrm{mgrms}$. - a total loss of $8 \mathrm{mgrms}$. Another wire, weighing $343 \mathrm{mgrms}$, was lkept moderately incandescent for nine consecutive hours, after which it weighed 301 mgrms, showing a total loss of 42 mgrms. A platinum wire twentythousandths of an inch in diameter was wound in the form of a spiral one-eighth of an inch in diameter and one-half an inch in length. The two ends of the spiral were secured to clamping posts, and the whole apparatus was covered with a glass shade $2 \frac{1}{2}$ inches in diameter and 3 inches high. Upon bringing the spiral to incandescence for twenty minutes that part of the globe in line with the sides of the spiral became slightly darkened in five hours the deposit became so thick that the incandescent spiral could not be seen through the deposit. This film, which was most perfect, consisted of platinum, and I have no doubt but that large plates of glass might be coated economically by placing them on each side of a large sheet of platinum, kept incandescen t by the electric current loss. in weight, together with the deposit upon the glass, presented a very serious obstacle to the use of metallic wires for giving light by incandescence, but this was easily surmounted after the cause was ascertained. I coated the wire forming the spiral with the oxide of magnesium, by (lusting upon it finely powdered acetate of magnesium: while incandescent the salt was decomposed by the heat, and there remained a strongly adherent coating of the oxide. This spiral so coated was covered with a glass shade, and brought to incandescence for several minutes ; but instead of a deposit of platinum upon the glass, there was a deposit of the oxide of magnesia. From this and other experiments I became convinced that this effect was due to the washing action of the air upon the spiral ; that the loss of weight in and the coloration of the hydrogen flame were also due to the wearing away of the surface of the platina to the attrition produced by the impact of the stream of gases upon the highly incandescent surface, and not to volatilisation, as commonly understood; and I venture to say, although I have not tried the experiment, that metallic sodium cannot be vola. tilised in high vacua by the heat derived from incandescent platinum; any effect that may be produced will be due to the washing action of the residual air. After the experiment last described I placed a spiral of platinum in the receiver of a common air-pump, and arranged it in such a manner that the current could pass through it, while the receiver was exhausted. At a pressure of 2 millimetres the spiral was kept at incan. descence for two hours before the deposit was sufficient to become visible. In another experiment, at a higher exhaustion, it required five hours before a deposit became visible. In a sealed glass bulb, exhausted by a. Sprengel pump to a point where a quarter of an inch spark from an induction-coil would not pass between points $\mathrm{I}$ millimetre apart, was placed a spiral, the connecting wires passing through the glass. This spiral has been kept at the most dazzling incandescence for hours without the slightest deposit becoming visible.

I will now describe other and far more important phenomena observed in my experiments. If a short length of platinum wir. one-thousandth of an inch in diameter be held in the flame of a Bunsen burner, at some part it will fuse, and a piece of the wire will be bent at an angle by the action of the globule of melted platinum; in some cases there are several globules formed simultaneously, and the wire assumes a zigzag shape. With a wire four-thousandths of an inch in diameter this effecs does not take place, as the temperature cannot be raised to equal that of the smaller wire, owing to the increased radiating surface and mass. After heating if the wire be examined under a microscope, that part of the surface which has been incandescent will be found covered with innumerable cracks. If the wire be placed between clamping posts, and heated to incandescence for twenty minutes, by the passage of an electric current, the cracks will: so enlarged as to be seen with the naked eye; the wire, under the microscope, presents a shrunken appearance, and is full of deep cracks. If the current is continued for several hours these effects will so increase that the wire will fall to pieces. This disintegration has been noticed in platina long subjected to the action of a flame by Prof. John W. Draper. The failure of the process of lighting invented by the French chemist Tessie du Motay, who raised sheets of platinum to incandescence by 